\title{
Prediction of patients with a tumor proportion score $>50 \%$ who do not respond to first-line monotherapy with pembrolizumab
}

Mitsunori Morita ${ }^{1 *}$ (D, Motohiro Tamiya ${ }^{2}$, Daichi Fujimoto ${ }^{3}$, Akihiro Tamiya $^{4}$, Hidekazu Suzuki ${ }^{5}$, Katsuya Hirano $^{6}$, Yasushi Fukuda ${ }^{7}$, Toshihide Yokoyama ${ }^{7}$, Ryota Kominami ${ }^{8}$, Masaki Kanazu ${ }^{9}$, Junji Uchida ${ }^{10}$, Satoshi Hara ${ }^{11}$, Shuji Yamashita ${ }^{1}$ and Hiromi Tomioka'

\begin{abstract}
Background: Pembrolizumab is effective as first-line therapy against advanced non-small cell lung cancer (NSCLC) in patients with programmed death ligand-1 (PD-L1) expression levels $\geq 50 \%$ [1]. However, it is not effective in all patients, and the factors predicting responses among this population remain unknown.

Methods: We retrospectively analyzed patients with NSCLC and a PD-L1 tumor proportion score (TPS) > 50\%, who received first-line monotherapy with pembrolizumab from February 1, 2017 to April 30, 2018. The study included 11 hospitals, which participated in the Hanshin Oncology clinical Problem Evaluation group (HOPE). We analyzed the differences between responders and non-responders in terms of age, sex, performance status score, degree of progression, histological type, smoking history, expression of PD-L1, use of steroids prior to treatment, metastasis site, and laboratory data.

Results: A total of 205 patients were included in this study. Of those, 108 patients exhibiting complete or partial response were defined as responders. Those exhibiting progressive disease $(N=52)$ were defined as non-responders. In the univariate analysis, Eastern Cooperative Oncology Group performance status score $\geq 2(p=0.0832)$, stage IV disease or recurrence $(p=0.0487)$, PD-L1 TPS 50-89\% ( $p=0.0657)$, use of steroids prior to the administration of pembrolizumab ( $p=0.0243)$, malignant pleural effusion ( $p=0.0032)$, and baseline C-reactive protein (CRP) levels $>1.0 \mathrm{mg} / \mathrm{dL}(p=0.0390)$ were significantly associated with non-response to treatment.

In the multivariate analysis, use of steroids prior to the administration of pembrolizumab (odds ratio [OR]: 5.86; $95 \%$ confidence interval [CI]: 1.32-31.8; $p=0.0200$ ), malignant pleural effusion (OR: 2.68; 95\% Cl: 1.15-6.35; $p=0.0228$ ), and baseline CRP $>1.0$ $\mathrm{mg} / \mathrm{dL}$ (OR: $2.17 ; 95 \% \mathrm{Cl}: 1.03-4.68 ; p=0.0402)$ were significantly associated with non-response to treatment.
\end{abstract}

Conclusion: In real-world patients with NSCLC and a PD-L1 TPS $\geq 50 \%$, use of steroids prior to treatment, malignant pleural effusion, and baseline CRP levels $>1.0 \mathrm{mg} / \mathrm{dL}$ reduced the response of first-line monotherapy with pembrolizumab.

Keywords: Non-small cell lung cancer, Pembrolizumab, First-line therapy, Efficacy, Programmed death ligand-1

\footnotetext{
*Correspondence: tamo.morita1123@gmail.com

'Department of Respiratory Medicine, Kobe City Medical Center West

Hospital, 2-4, Ichiban-cho, Nagata-ku, Kobe-shi, Hyogo 653-0013, Japan

Full list of author information is available at the end of the article
}

(c) The Author(s). 2020 Open Access This article is distributed under the terms of the Creative Commons Attribution 4.0 International License (http://creativecommons.org/licenses/by/4.0/), which permits unrestricted use, distribution, and reproduction in any medium, provided you give appropriate credit to the original author(s) and the source, provide a link to the Creative Commons license, and indicate if changes were made. The Creative Commons Public Domain Dedication waiver (http://creativecommons.org/publicdomain/zero/1.0/) applies to the data made available in this article, unless otherwise stated. 


\section{Background}

Non-small-cell lung cancer (NSCLC) is the leading cause of cancer-related death worldwide, and the outcomes for patients with NSCLC are currently poor [2] . However, the development of programmed cell death (PD)-1 immune checkpoint inhibitors (ICIs) has improved treatment outcomes for NSCLC [3-5]. The results of the international, randomized, open-label, phase 3 KEYNOTE-024 trial showed that, in patients with advanced NSCLC and programmed cell death ligand-1 (PD-L1) expression levels $\geq 50 \%$ in tumor cells, treatment with pembrolizumab was associated with significantly longer progression-free survival (PFS) and overall survival (OS), and fewer adverse events versus platinum-based chemotherapy $[1,6]$.

Pembrolizumab has dramatically changed the first-line standard therapy for patients with high levels $(\geq 50 \%)$ of PD-L1 expression in daily clinical practice. Furthermore, in the KEYNOTE-024 trial, the objective response rate in the pembrolizumab group was $44.8 \%$. Numerous cases have demonstrated the significant therapeutic effect of pembrolizumab in the real-world setting. However, in a number of cases, treatment with pembrolizumab is ineffective. Using real-world data obtained from multiple institutions (including city hospitals), in the present study, we examined patients who did not respond to monotherapy with pembrolizumab despite exhibiting high levels of PD-L1 expression.

\section{Methods}

This was a multicenter, observational, retrospective cohort study involving patients with NSCLC who received first-line monotherapy with pembrolizumab.

We investigated a total of 213 patients from 11 hospitals participating in the Hanshin Oncology clinical Problem Evaluation group (HOPE) from February 1, 2017 to April 30, 2018.
The effect of the treatment was evaluated according to the Response Evaluation Criteria in Solid Tumors: Revised (RECIST) guideline (version 1.1). Eight patients were excluded for the following reasons: changing hospital $(N=3)$, death prior to the evaluation of the treatment effect $(N=2)$, drug-induced lung injury and switch to a subsequent treatment $(\mathrm{N}=2)$, recurrence of brain metastasis, and use of radiotherapy $(N=1)$. Therefore, a total of 205 patients were included in the analysis. Patients were followed-up for disease status until August 31, 2018.

\section{Statistical analysis}

For all statistical analyses, the $\mathrm{JMP}^{\circledR}$ statistical software program (12th version; SAS Institute Inc., Cary, NC, USA) was used. Categorical variables were analyzed using the $\chi^{2}$ test or Fisher's exact test. A $p<0.05$ denoted statistically significant differences. Multivariate logistic regression analysis was performed by selecting factors with $p<0.10$ in the univariate analysis. The Kaplan-Meier method was used to produce the survival curve.

This study was approved by the institutional review board of each participating hospital, and is registered with the UMIN (University Hospital Medical Information Network in Japan; number 000032470).

\section{Results}

\section{Patient characteristics}

The clinical characteristics of the 205 patients are shown in Table 1. The median age at the time of treatment with pembrolizumab was 70 years (range: 44-91 years). The majority of the patients $(N=170 ; 82.9 \%)$ were males, and 169 patients (82.4\%) had an Eastern Cooperative Oncology Group performance status (ECOG PS) of $0-1$. Most patients $(N=168$; $82.0 \%$ ) had stage IV disease or recurrence; however, a number of patients with stage II and III disease were inoperative and

Table 1 Patient characteristics at baseline

\begin{tabular}{|c|c|}
\hline Characteristics & Patients $(N=205)$ \\
\hline Median age (range), years & $70(44-91)$ \\
\hline Gender: male / female & $170 / 35$ \\
\hline ECOG PS score: $0 / 1 / 2 / 3 / 4$ & $49 / 120 / 29 / 6 / 1$ \\
\hline Stage: II / III / IV recurrence & $3 / 34 / 130 / 38$ \\
\hline Histological types: ADC / SCC / NSCLC-NOS / other & $123 / 54 / 22 / 6^{a}$ \\
\hline EGFR mutation: positive / negative / unknown & $6^{\mathrm{b}} / 171 / 28$ \\
\hline Smoking history: ever / never / unknown & $182 / 19 / 4$ \\
\hline PD-L1: 50-89\% / 90-100\% & $138 / 67$ \\
\hline Steroid use: yes / no & $13 / 192$ \\
\hline Best response: CR / PR / SD / PD & $3 / 105 / 45 / 52$ \\
\hline
\end{tabular}

Abbreviations: ECOG PS, Eastern Cooperative Oncology Group performance status, ADC adenocarcinoma, SCC squamous cell carcinoma; NSCLC-NOS non-small cell carcinoma -not otherwise specified, EGFR, epidermal growth factor receptor, $P D$ - $L 1$ programmed cell death ligand 1, $C R$ complete response, $P R$ partial response, $S D$ stable disease, $P D$ progressive disease, Ex19del exon 19 deletion

a pleomorphic carcinoma: four cases; spindle cell carcinoma: one case; large cell carcinoma: one case

bx19del: two cases; Ex19del + T790 M: one case; G719A: two cases; G719C: one case 
unable to receive radiation therapy. The tissue types were squamous cell carcinoma $(N=54 ; 26.3 \%)$, adenocarcinoma $(N=123 ; 60.0 \%)$, NSCLC not otherwise specified $(N=22$; $10.7 \%)$, pleomorphic carcinoma $(N=4 ; 2.0 \%)$, spindle cell carcinoma $(N=1 ; 0.5 \%)$, and large cell carcinoma $(\mathrm{N}=1 ; 0.5 \%)$. Six patients were epidermal growth factor receptor mutationpositive: exon 19 deletion $(\mathrm{N}=2)$; exon 19 deletion+T790 M $(\mathrm{N}=1)$; G719A $(\mathrm{N}=2)$; and $\mathrm{G719C}(\mathrm{N}=1)$. A total of 182 patients (88.8\%) had smoking history. There were 138 patients (67.3\%) with a tumor proportion score (TPS) of 50-90\%, and 67 patients $(32.7 \%)$ with a TPS of $90-100 \%$. Of note, there were 13 patients $(6.3 \%)$ who received steroids prior to the initiation of treatment with pembrolizumab (Table 1).

\section{Difference in treatment effectiveness}

Complete response (CR), partial response (PR), stable disease (SD), or progressive disease (PD) was observed in three, 105, 45, and 52 patients, respectively. The response rate was $52.7 \%$ and the disease control rate was $74.6 \%$.

In this study, we classified the patients into two groups: responders (108 patients exhibiting CR or PR) and non-responders (52 patients exhibiting PD).

We compared the baseline characteristics of responders and non-responders in terms of age, sex, performance status score, degree of progression, histological type, smoking history, expression of PD-L1, use of steroids prior to treatment, metastasis site, and laboratory data.
In the univariate analysis, ECOG PS score $\geq 2$ ( $p=$ $0.0832)$, stage IV disease or recurrence ( $p=0.0487)$, PD-L1 TPS $50-89 \%$ ( $p=0.0657$ ), use of steroids prior to the administration of pembrolizumab $(p=0.0243)$, malignant pleural effusion $(p=0.0032)$, and baseline C-reactive protein (CRP) levels $>1.0 \mathrm{mg} / \mathrm{dL}(p=0.0390)$ were significantly associated with non-response to treatment (Table 2).

ECOG PS score $\geq 2$, stage IV disease or recurrence, a TPS of $50-90 \%$, use of steroids prior to treatment, the presence of pleural effusion, and baseline CRP levels > $1.0 \mathrm{mg} / \mathrm{dL}$ yielded a $p<0.10$ in the univariate analysis, and were included in the multivariate analysis.

In the multivariate analysis, use of steroids prior to the administration of pembrolizumab (odds ratio [OR]: 5.86; 95\% confidence interval [CI]: $1.32-31.8 ; p=0.0200)$, the presence of malignant pleural effusion (OR: 2.68; 95\% CI: $1.15-6.35 ; p=0.0228)$, and baseline CRP levels $>1.0$ mg/dL (OR: 2.17; 95\% CI: 1.03-4.68; $p=0.0402$ ) were significantly associated with non-response to treatment (Table 3).

We further analyzed 52 patients (non-responders) who presented PD after monotherapy with pembrolizumab. After the administration of pembrolizumab, the ECOG PS score decreased in 25 patients (48.1\%). Second-line treatment was administered in 35 patients (67.3\%); however, best supportive care was applied in 17 patients $(32.7 \%)$.

Table 2 Univariate analysis

\begin{tabular}{|c|c|c|c|}
\hline Factor & $\begin{array}{l}\text { Responder } \\
(N=108)\end{array}$ & $\begin{array}{l}\text { Non-responder } \\
(N=52)\end{array}$ & $\begin{array}{l}p \\
\text { value }\end{array}$ \\
\hline Age $>70$ years & $59(54.6)$ & $29(55.8)$ & 0.8920 \\
\hline Female sex & $16(14.8)$ & $10(19.2)$ & 0.4782 \\
\hline ECOG PS score $\geq 2$ & $15(13.9)$ & $13(25.0)$ & 0.0832 \\
\hline Stage: IV, recurrence & $81(75.0)$ & $46(88.5)$ & 0.0487 \\
\hline Squamous cell carcinoma & $29(26.9)$ & $15(28.9)$ & 0.7913 \\
\hline Never smoker & $9(8.3)$ & $5(9.6)$ & 0.7881 \\
\hline PD-L1 50-89\% & $65(60.2)$ & $39(75.0)$ & 0.0657 \\
\hline Steroid use & $3(2.8)$ & $6(11.5)$ & 0.0243 \\
\hline Metastasis Brain & $14(13.0)$ & $12(23.1)$ & 0.1043 \\
\hline Liver & $12(11.1)$ & $8(15.4)$ & 0.4439 \\
\hline Bone & $28(25.9)$ & $14(26.9)$ & 0.8932 \\
\hline Adrenal & $17(15.7)$ & $10(19.2)$ & 0.5809 \\
\hline Pulmonary & $31(28.7)$ & $20(38.5)$ & 0.2148 \\
\hline Pleural effusion & $17(15.7)$ & $19(36.5)$ & 0.0032 \\
\hline \multicolumn{4}{|l|}{ Laboratory data } \\
\hline Neutrophil-to-lymphocyte ratio $(<3)$ & $30(27.8)$ & $14(26.9)$ & 0.9097 \\
\hline C-reactive protein $(<1.0 \mathrm{mg} / \mathrm{dL})$ & $54(50.0)$ & $17(32.7)$ & 0.0390 \\
\hline Lactate dehydrogenase (<240 IU/L) & $79(73.2)$ & $33(63.5)$ & 0.2105 \\
\hline Albumin $(<3.5 \mathrm{~g} / \mathrm{dL})$ & $47(43.5)$ & $26(50.0)$ & 0.4407 \\
\hline
\end{tabular}


Table 3 Multivariate logistic regression analysis

\begin{tabular}{llll}
\hline Factor & Odds ratio & $95 \%$ confidence interval & $p$ value \\
\hline ECOG PS score $\geq 2$ & 1.44 & $0.57-3.59$ & 0.4366 \\
Stage: IV, recurrence & 1.65 & $0.61-5.02$ & 0.3357 \\
PD-L1 50-89\% & 1.91 & $0.88-4.30$ & 0.1011 \\
Steroid use & 5.86 & $1.32-31.8$ & 0.0200 \\
Metastasis pleural effusion & 2.68 & $1.15-6.35$ & 0.0228 \\
C-reaction protein $(<1.0 \mathrm{mg} / \mathrm{dl})$ & 2.17 & $1.03-4.68$ & 0.0402 \\
\hline
\end{tabular}

ECOG PS Eastern Cooperative Oncology Group performance status, PD-L1 programmed cell death ligand 1

Among those who received second-line treatment, 19 patients achieved PR, seven patients exhibited stable disease, and nine patients experienced PD. The median OS in nonresponders was 255 days with poor prognosis. (Fig. 1).

\section{Discussion}

Pembrolizumab has been shown to be effective as primary treatment in NSCLC patients with PD-L1 expression levels $\geq 50 \%$. However, it is not necessarily effective in all patients. Therefore, the prediction of non-response is of crucial importance in determining the most appropriate treatment regimen.

Based on the results of this retrospective cohort study, pleural effusion, baseline CRP levels $>1.0 \mathrm{mg} / \mathrm{dL}$, and use of steroids prior to treatment tended to reduce the effectiveness of first-line monotherapy with pembrolizumab.

Firstly, we investigated the association between the use of steroids and the effectiveness of pembrolizumab. Taniguchi et al. reported that, in patients treated with nivolumab, ECOG PS score $\geq 2$, use of steroids at baseline, and lactate dehydrogenase levels $>240 \mathrm{IU} / \mathrm{L}$ were significantly associated with poor PFS [7]. Arbor et al. reported that use of corticosteroids $(\geq 10 \mathrm{mg}$ prednisone or equivalent) at baseline was associated with poorer outcome in patients with NSCLC, who were treated with PD-(L)1 blockade [8]. These studies included patients with any PD-L1 status and lines of therapy.

This study investigated only treatment-naive patients with high expression levels of PD-L1. Consistent with previous reports, treatment with the ICI tended to be less effective in patients who had received prior treatment with steroids.

Secondly, we investigated the association between CRP and response to ICI. Oya et al. reported that, among patients treated with nivolumab, the objective response rate in those with elevated CRP levels $(\geq 1.0 \mathrm{mg} / \mathrm{dL})$ was significantly worse than that reported in patients without elevated CRP levels $(<1.0 \mathrm{mg} / \mathrm{dL})$ [9]. In addition, Inoue et al. reported that, among patients treated with nivolumab, a CRP-toalbumin ratio $>0.3$ was associated with early death mainly due to PD and/or the occurrence of immune-related adverse events [10]. Although these are reports of nivolumab, in the present study, pembrolizumab (another PD-1 inhibitor) demonstrated similar findings.

Thirdly, to the best of our knowledge, few studies have assessed the therapeutic effects of ICIs in patients complicated

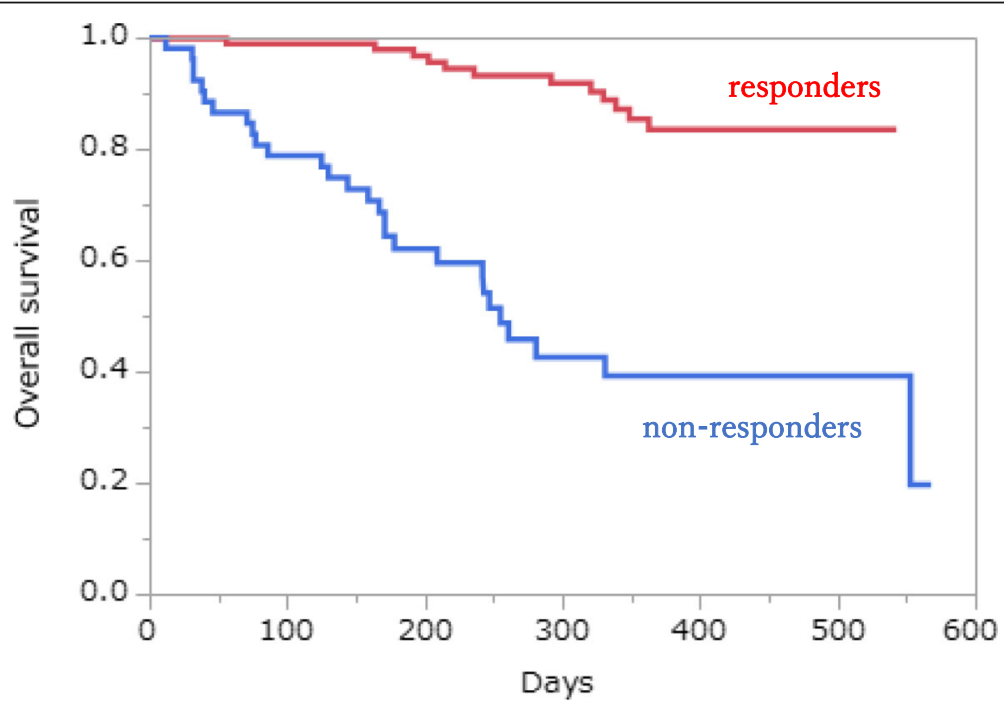

Fig. 1 Overall survival in responders and non-responders who received pembrolizumab as first-line therapy 
with pleural effusion. Kang et al. reported the response rate in advanced NSCLC patients treated with PD1/PD-L1 inhibitors. The results showed that the response rate was markedly lower in patients with pleural or pericardial metastasis than that observed in those without pleural or pericardial metastasis [11]. Shibaki et al. reported that the presence of malignant pleural effusion was an independent negative predictor affecting PFS and OS, regardless of the presence of positive PD-L1 expression [12]. Furthermore, these studies showed that ICI monotherapy tended to be less effective in patients with pleural effusion. However, the mechanism responsible for the low efficacy of ICIs observed in patients with pleural effusion remains to be elucidated [11, 12].

The KEYNOTE-189 study investigated patients with previously untreated metastatic non-squamous NSCLC without epidermal growth factor receptor or anaplastic lymphoma kinase mutations. In that study, the addition of pembrolizumab to standard chemotherapy (i.e., pemetrexed and a platinum-based drug) resulted in significantly longer OS and PFS versus chemotherapy alone [13]. Moreover, the KEYNOTE-407 study examined patients with previously untreated metastatic, squamous NSCLC. In that study, the addition of pembrolizumab to chemotherapy (i.e., carboplatin plus paclitaxel or nabpaclitaxel) resulted in significantly longer OS and PFS versus chemotherapy alone [14].

In daily practice, it may be difficult to decide whether to choose pembrolizumab monotherapy or combination therapy (i.e., platinum-based chemotherapy and ICI), especially for patients with high PD-L1 expression. Our study may be helpful in selecting treatments among many treatment options. Further studies investigating the use of ICI monotherapy or combination therapy from an efficacy, safety, and medical cost perspective are warranted.

The present study was characterized by several limitations. Firstly, this was a retrospective study. Secondly, treatment effectiveness was evaluated based on the routine practice of each physician. Thirdly, examination of pleural effusion was not performed in all patients. The presence of malignant pleural effusion was defined as not only cytology but also clinical diagnosis such as clinical course and imaging findings. Fourthly, we have not considered details about previous steroid use such as the reason, duration, relation with lung cancer and when the patients received steroid.

\section{Conclusions}

Our study may help to predict patients in whom firstline monotherapy with pembrolizumab is not effective despite the presence of a PD-L1 TPS $\geq 50 \%$. In particular, the use of steroids prior to the administration of pembrolizumab, the presence of malignant pleural effusion, and baseline CRP levels $>1.0 \mathrm{mg} / \mathrm{dL}$ tended to reduce the response of ICI monotherapy.

\section{Additional file}

Additional file 1. Institutional Review Board in Osaka International Cancer Institute (approval No.1802199367) and each institution.

\section{Abbreviations}

CR: Complete response; CRP: C-reactive protein; ECOG PS: Eastern Cooperative Oncology Group performance status; ICl: Immune checkpoint inhibitor; NSCLC: Non-small cell lung cancer; OS: Overall survival; PD: Progressive disease; PD-L1: Programmed death ligand-1; PFS: Progression-free survival; PR: Partial response; RECIST: Response Evaluation Criteria in Solid Tumors; SD: Stable disease; TPS: Tumor proportion score

\section{Acknowledgements}

We are grateful to all patients and investigators who participated in this study.

\section{Authors' contributions}

MT and DF were involved in study concepts and design. MM, MT, DF, AT, HS, KH, YF, TY, RK, MK, JU, SH, and SY were involved in data acquisition. MM and HTperformed data analysis and wrote the manuscript. All authors approved the final manuscript.

\section{Funding}

This research did not receive any specific grant from funding agencies in the public, commercial, or not-for-profit sectors.

\section{Availability of data and materials}

The datasets generated during and/or analyzed during the current study are available from the corresponding author on reasonable request.

\section{Ethics approval and consent to participate}

All procedures performed in studies involving human participants were in accordance with the ethical standards of the institutional and/or national research committee and with the 1964 Helsinki declaration and its later amendments or comparable ethical standards. This retrospective study was approved by the Institutional Review Board in Osaka International Cancer Institute (approval No.1802199367) and each institution (Additional file 1). For this type of study, formal consent is not required.

\section{Consent for publication}

Not applicable.

\section{Competing interests}

Drs. M.Tamiya, A.Tamiya, Fujimoto, and Hirano have received lecture fees from Taiho Pharmaceutical Co., Ltd. (Tokyo, Japan) and Merck Sharp \& Dohme, Corp. (Tokyo, Japan). Drs. Yokoyama has received lecture fees from Taiho Pharmaceutical Co., Ltd. (Tokyo, Japan). Drs. Kanazu has received lecture fees from Merck Sharp \& Dohme, Corp. (Tokyo, Japan). The remaining authors have no conflicts of interest to declare.

\footnotetext{
Author details

${ }^{1}$ Department of Respiratory Medicine, Kobe City Medical Center West Hospital, 2-4, Ichiban-cho, Nagata-ku, Kobe-shi, Hyogo 653-0013, Japan. ${ }^{2}$ Department of Thoracic Oncology, Osaka International Cancer Institute, 3-1-69 Otemae, Chuo-ku, Osaka 541-8567, Japan. ${ }^{3}$ Department of Respiratory Medicine, Kobe City Medical Center General Hospital, 2-1-1

Minatojimaminamimachi, Chuo-ku, Kobe-shi, Hyogo 650-0047, Japan. ${ }^{4}$ Department of Internal Medicine, National Hospital Organization Kinki-Chuo Chest Medical Center, 1180, Nagasone-cho, Kita-ku, Sakai-shi, Osaka 591-8555, Japan. ${ }^{5}$ Department of Thoracic Oncology, Osaka Habikino Medical Center, 3-7-1, Habikino, Habikino-shi, Osaka 583-8588, Japan. 'Department of Respiratory Medicine, Hyogo Prefectural Amagasaki General Medical Center, 2-17-77, Higashi-Naniwa-Cho, Amagasaki-shi, Hyogo 660-8550, Japan. ${ }^{7}$ Department of Respiratory Medicine, Kurashiki Central Hospital, 1-1-1, Miwa, Kurashiki-shi, Okayama 710-8602, Japan. ${ }^{8}$ Department of Respiratory Medicine, National Hospital Organization Himeji Medical Center, 68, Honmachi, Himeji-shi, Hyogo 670-8520, Japan. ${ }^{9}$ Department of Thoracic Oncology, National Hospital Organization Osaka Toneyama Medical Center, 5-1-1, Toneyama, Toyonaka-shi, Osaka 560-0045, Japan. ${ }^{10}$ Department of Respiratory Medicine, Osaka General Medical Center, 3-1-56, Bandai-Higashi, Sumiyoshi-ku, Osaka 558-8558, Japan. ${ }^{11}$ Department of Respiratory Medicine, Itami City Hospital, 1-100, Koyaike, Itami-shi, Hyogo 664-8540, Japan.
} 
Received: 29 September 2019 Accepted: 27 January 2020

Published online: 03 February 2020

\section{References}

1. Reck M, Rodríguez-Abreu D, Robinson AG, Hui R, Csőszi T, Fülöp A, et al. Pembrolizumab versus chemotherapy for PD-L1-positive non-small-cell lung Cancer. N Engl J Med. 2016;375(19):1823-33.

2. Siegel RL, Miller KD, Jemal A. Cancer statistics, 2017. CA Cancer J Clin. 2017; 67(1):7-30.

3. Brahmer J, Reckamp KL, Baas P, Crinò L, Eberhardt WE, Poddubskaya E, et al. Nivolumab versus Docetaxel in advanced squamous-cell non-small-cell lung Cancer. N Engl J Med. 2015;373(2):123-35.

4. Borghaei H, Paz-Ares L, Horn L, Spigel DR, Steins M, Ready NE, et al. Nivolumab versus Docetaxel in advanced nonsquamous non-small-cell lung Cancer. N Engl J Med. 2015;373(17):1627-39.

5. Herbst RS, Baas P, Kim DW, Felip E, Pérez-Gracia JL, Han JY, et al. Pembrolizumab versus docetaxel for previously treated, PD-L1-positive, advanced non-small-cell lung cancer (KEYNOTE-010): a randomised controlled trial. Lancet. 2016:387(10027):1540-50.

6. Reck M, Rodríguez-Abreu D, Robinson AG, Hui R, Csőszi T, Fülöp A, et al. Updated analysis of KEYNOTE-024: Pembrolizumab versus platinum-based chemotherapy for advanced non-small-cell lung Cancer with PD-L1 tumor proportion score of 50\% or greater. J Clin Oncol. 2019;37(7):537-46.

7. Taniguchi Y, Tamiya A, Isa SI, Nakahama K, Okishio K, Shiroyama T, et al. Predictive factors for poor progression-free survival in patients with non-small cell lung Cancer treated with Nivolumab. Anticancer Res. 2017;37(10):5857-62.

8. Arbour KC, Mezquita L, Long N, Rizvi H, Auclin E, Ni A, et al. Impact of baseline steroids on efficacy of programmed cell Death-1 and programmed death-ligand 1 blockade in patients with non-small-cell lung Cancer. J Clin Oncol. 2018;36(28):2872-8.

9. Oya Y, Yoshida T, Kuroda H, Mikubo M, Kondo C, Shimizu J, et al. Predictive clinical parameters for the response of nivolumab in pretreated advanced non-small-cell lung cancer. Oncotarget. 2017;8(61):103117-28.

10. Inoue T, Tamiya M, Tamiya A, Nakahama K, Taniguchi $Y$, Shiroyama T, et al. Analysis of early death in Japanese patients with advanced non-small-cell lung Cancer treated with Nivolumab. Clin Lung Cancer. 2018;19(2):e171-6.

11. Kang DH, Chung C, Kim JO, Jung SS, Park HS, Park DI, et al. Pleural or pericardial metastasis: a significant factor affecting efficacy and adverse events in lung cancer patients treated with PD-1/PD-L1 inhibitors. Thorac Cancer. 2018;9(11):1500-8.

12. Shibaki R, Murakami S, Shinno Y, Matsumoto Y, Goto Y, Kanda S, et al. Malignant pleural effusion as a predictor of the efficacy of anti-PD-1 antibody in patients with non-small cell lung cancer. Thorac Cancer. 2019;10(4):815-22.

13. Gandhi L, Rodríguez-Abreu D, Gadgeel S, Esteban E, Felip E, De Angelis F, et al. Pembrolizumab plus chemotherapy in metastatic non-small-cell lung Cancer. N Engl J Med. 2018;378(22):2078-92.

14. Paz-Ares L, Luft A, Vicente $D$, Tafreshi $A$, Gümüş $M$, Mazières J, et al. Pembrolizumab plus chemotherapy for squamous non-small-cell lung Cancer. N Engl J Med. 2018;379(21):2040-51.

\section{Publisher's Note}

Springer Nature remains neutral with regard to jurisdictional claims in published maps and institutional affiliations.

Ready to submit your research? Choose BMC and benefit from:

- fast, convenient online submission

- thorough peer review by experienced researchers in your field

- rapid publication on acceptance

- support for research data, including large and complex data types

- gold Open Access which fosters wider collaboration and increased citations

- maximum visibility for your research: over $100 \mathrm{M}$ website views per year

At BMC, research is always in progress.

Learn more biomedcentral.com/submissions 